\title{
Cooperative Intelligence in Roundabout Intersections Using Hierarchical Fuzzy Behavior Calculation of Vehicle Speed Profile
}

\author{
Ivan Bosankić ${ }^{1}$ and Lejla Banjanović Mehmedović ${ }^{2}$ \\ ${ }^{1}$ IVL, 75000 Tuzla, Bosnia and Herzegovina \\ ${ }^{2}$ University of Tuzla, Faculty of Electrical Engineering, 75000 Tuzla, Bosnia and Herzegovina
}

\begin{abstract}
In this paper, a new fuzzy-behavior-based algorithm for roundabout intersection management is presented. The algorithm employs cooperative intelligence and includes intelligent vehicles and infrastructure to calculate speed profiles for different vehicles, in order to achieve more comfortable driving profiles, as well to reduce congestion and $\mathrm{CO}_{2}$ emissions. The algorithm uses adaptive spatio-temporal reservation technique and was tested in MATLAB/Simulink environment. The algorithm is designed to function in different scenarios with both cooperative and non-cooperative vehicles, as well as optional intersection infrastructure. Results have show that using the proposed algorithm different vehicle communication types can be successfully combined in order to increase traffic flow through roundabout intersections.
\end{abstract}

\section{Introduction}

Roundabout intersections have recently become very popular, since they reduce number of conflict points which is characteristic for classic intersections, reduce driving speeds and increase driver attention [1]. The traffic flow can be as high as 70.000 vehicles per day. In such roundabouts, waiting times are high, and collisions happen often [2]. When traffic is heavy, waiting time is a significant problem. Normal approach would be building elevated road lanes, but often free space is an issue. Possible solutions include installation of traffic lights at roundabout entry, which can decrease waiting times during increased traffic flow times [3], especially if it is optimized [4].

Modern approaches, such as flower and turbo roundabouts present recent solutions that improve road safety and reduce number of collisions, but waiting time when traffic flow is above 1500 vehicles per hour increases more than five times when compared to 1000 vehicles per hour [5]. Increased capacity also increases pollutant emissions [6].

\subsection{State of the art}

With introduction of the smart vehicles, alternative methods for roundabout management have emerged. Although it has been considered for a long time that traffic lights are only way to achieve efficient traffic control actuator function [7], with increased development of self driving vehicles, it becomes clear that a new approach to enforce traffic control on intersections can be expected in the future.
V2V and V2I communications represent an important role in modern Intelligent Transportation System (ITS), and they can be used to exchange inter-vehicular signals, therefore avoiding classic traffic lights. New models implement Virtual Traffic Lights (VTL) [8] as method that allows each smart car to receive its individual traffic signal, therefore acquiring better control and throughput. Using virtual traffic lights it is possible to increase average speed up to $26 \%$ in low and up to $41 \%$ in high density traffic, while reducing $\mathrm{CO}_{2}$ emissions up to $18 \%$ [9].

This system can further be improved by advanced algorithms for intersection management, which also calculates speed profiles for each vehicle that passes through roundabout, spatio-temporal intersection management etc.

There are many recent works that address intersection, but they rarely address roundabout intersections. Basically, there are approaches that employ centralized control, by implementing intersection management unit which collects data from all participating vehicles and then calculates best speed profiles and assigns priorities: [10] implements supervisor which receives state information from the cars and then estimates possible collisions and sends overriding signal if necessary, [11] presents reservation-based intersection control algorithm. Others approaches include distributed intersection management, e.g. [12] assigns priorities to vehicles while introducing various $\mathrm{V} 2 \mathrm{~V}$ protocols.

Evolutionary algorithms (GA and SFLA) via V2V communication are used in [13], while [14] employs fuzzy logic for intersection detection and crossing. In [15] mutual exclusion algorithm is introduced, which is 
organized via V2V (distributed, without need of intersection controller). Some papers use linear programming algorithms, e.g. [16] uses job-shop scheduling. Centralized V2I coordination that employs Active set method and Interior point method for optimization is used in [17]. In [18] and [19] genetic optimization of fuzzy controller are used to achieve better results. Agent based approaches are often used, e.g. zoning for trajectory adjustment in [20]. In [21] a hybrid automaton for designing intersection management algorithm is used.

Since vehicles without communication capabilities cannot be excluded, [19], [22] and [23] suggest several algorithms both in roundabouts and classical intersections, based on spatio-temporal reservation technique.

In this paper, a new fuzzy-behavior-based algorithm for roundabout intersection management is presented. The advantage of our Cooperative Intelligence approach is that various different vehicle communication types combinations of cooperative and non-cooperative vehicles as well as possibility of faulty or missing infrastructure controller can be modeled and examined. Our algorithms increase the traffic flow and reduce the travel time through a roundabout intersection. In Section 2, Roundabout intersection model used for testing the proposed algorithm is explained, as well as concept of spatio-temporal reservation and hierarchical fuzzy behavior algorithm. In Section 3, cooperative intelligence principle is explained and several different cases are analyzed, while Section 4 contains research discussion. The fifth section is the conclusion and future work.

\section{Roundabout intersection model}

Roundabout intersection (RI), analyzed in this work consists of 5 of branches, marked with letters A-E, as shown in Fig. 1. This model can be extended to any finite number of branches. Each branch has entry and exit lane (indexed 1 and 2), and each part is divided in equal segments which are numbered and used for spatiotemporal reservation.

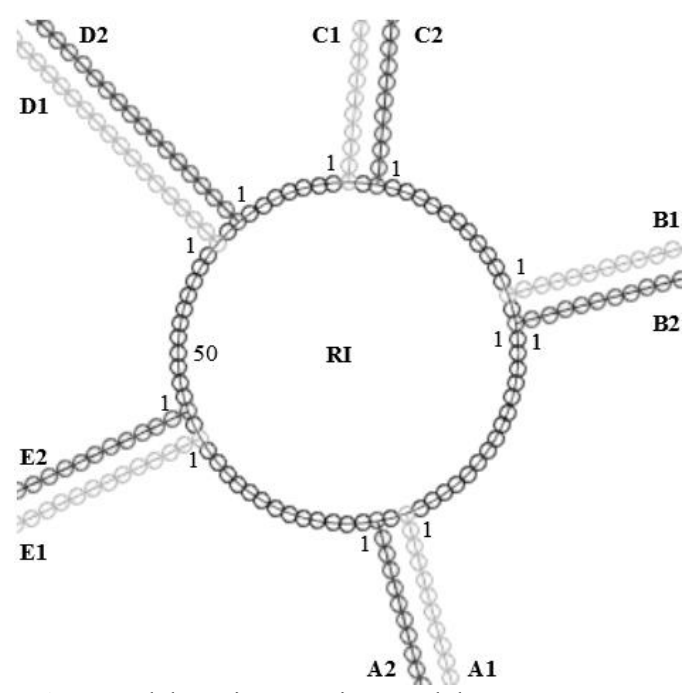

Figure 1. Roundabout intersection model.
Intersection may have intersection management computer (IMC), which collects data about vehicles inside roundabout and coordinates vehicles via V2I communication.

There are two types of vehicles in this model: cooperative (intelligent) and non-cooperative (ordinary) vehicles. Vehicles can exchange information between each other (V2V) or with IMC (V2I). It is supposed that IMC, if exists and functions correctly, can obtain location and speed data for every non-cooperative vehicle in the intersection.

Proposed algorithm includes fuzzy hierarchical behavior coordination of speed profiles for each vehicle.

\subsection{Reservation array}

Each cooperative vehicle forms and updates spatiotemporal reservation array. Roundabout is divided in finite number of discrete segments. In order to provide intersection management, this array must be filled for each vehicle. Cooperative autonomous vehicles maintain their own reservation array, since they have complete information about entrance and desired exit from the roundabout. This array is then exchanged with other vehicles via V2V and with IMC, via V2I. For other vehicles, IMC must use its sensors to store information about the vehicles. IMC forms reservation array based on measured speed, but here all exits must be considered, since real trajectory of the vehicle is unknown. IMC has no information on exit branch of non-cooperative vehicle, so it has to fill all possible exiting branches for this vehicle. Example of spatial reservation arrays for two vehicles is shown in Fig. 2. As we can see here, segments 4-16 of Roundabout intersection ring are both occupied by both vehicles, but it does not mean that vehicles occupy them at the same time. Spatio-temporal reservation is analyzed in Section III.

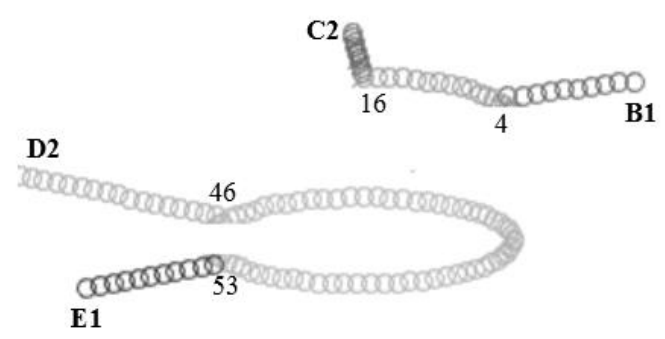

Figure 2. Example of spatial reservation arrays for two vehicles.

\subsection{Fuzzy spatial speed profile}

Speed profile on an intersection depends on various parameters: speed limit, roundabout radius, entry and exit road angles, vehicle limits and dynamics, lane widths, lane speeds etc. In order to ease calculation of reservation array, Fuzzy spatial speed profile block is proposed. It provides intuitive and easy way to determine vehicle speed, based on available parameters. Example of one speed profile is shown in Fig. 3. 


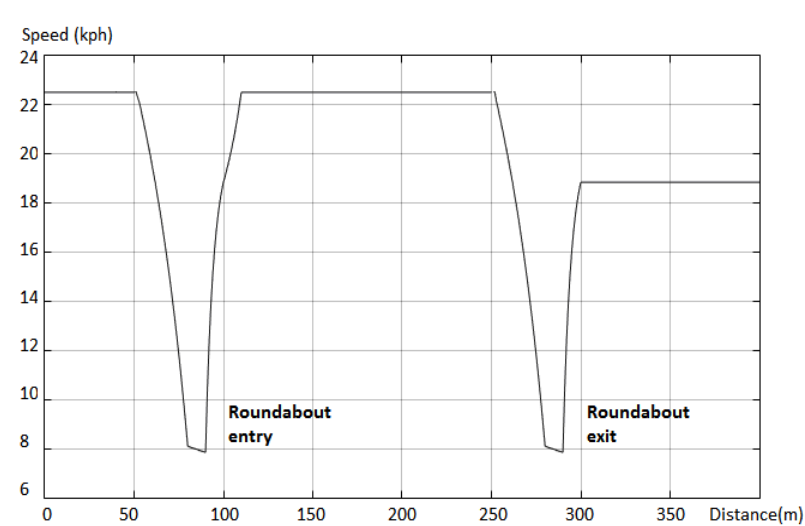

Figure 3. Example of fuzzy speed profile in roundabout intersection

\subsection{Fuzzy hierarchical speed behavior profile}

To implement proposed speed calculation algorithm, a hierarchical fuzzy-based speed calculation is used. The vehicle speed is influenced by three behaviors:

- Normal speed profile behavior is used to calculate normal speeds based on vehicle entry and exit point, as well as speed limits inside the roundabout. Spatial speed profile from Section 2.1 is converted in time domain so it can be used in hierarchical fuzzy

- Collision avoidance behavior is used to avoid collisions in case vehicles unexpectedly change its course, mostly as interaction with non-cooperative vehicles. It is implemented as simple reactive algorithm, which utilizes sensor inputs and IMC warnings to make corrections to Normal speed profile. Output of this block is speed change and can only be negative.

- Cooperative intelligence behavior is used to coordinate vehicles, in order to avoid congestions. Due to its nature, it can provide both positive and negative speed change commands as output. Cooperative algorithm is explained in detail in Section 3.

All proposed behaviors are fused in Hierarchical fuzzy speed profile block, as shown in Fig. 4.

Hierarchical fuzzy speed profile block forms corrected speed profile for an autonomous vehicle, based on sensor and cooperative corrections to Normal speed profile, Fig. 5. It is used to calculate updated reservation array, and if it overlaps with reservation arrays from other vehicles, additional cooperative actions need to be employed.

\subsection{MATLAB/Simulink model}

Since reservation arrays were needed to be updated in regular discrete intervals, combination of MATLAB script and Simulink models was used in simulation. In order to convert Normal speed profile from spatial to time domain, speed profile calculation was formed in separate model. Hierarchical fuzzy behavior speed profile is obtained by new simulation in regular intervals of $1 \mathrm{~s}$, Fig. 6. Reservation array was then recalculated based on speed profile and compared with other profiles.

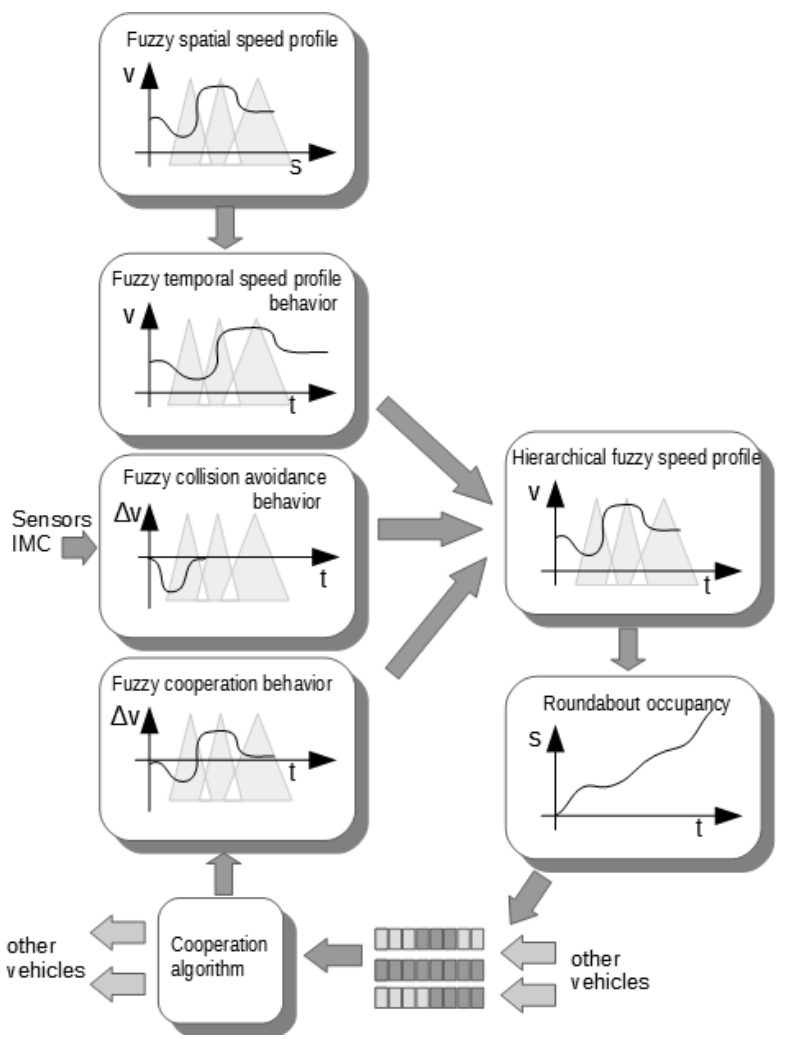

Figure 4. Hierarchical fuzzy vehicle speed model.

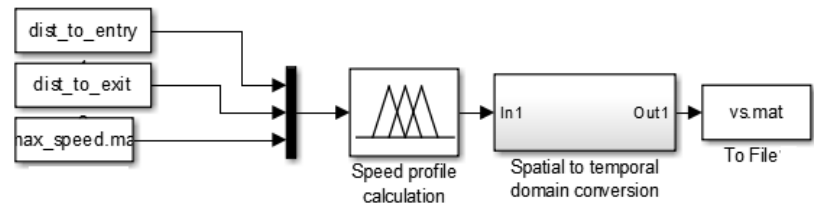

Figure 5. Simulink model for normal speed profile.

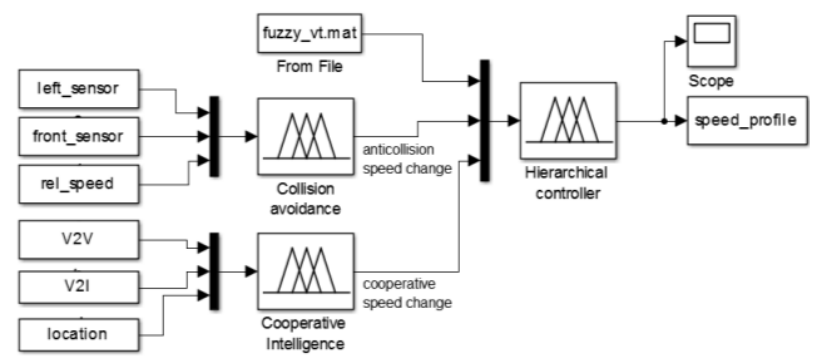

Figure 6. Hierarchical fuzzy vehicle speed model.

\section{Cooperative intelligence}

A proposed coordination algorithm aims to cover different cases of cooperative and non-cooperative vehicles, with or without intersection management controller (e.g. if IMC is broken).

Base for coordination algorithm are reservation arrays. Obtained reservation arrays for all vehicles are compared in order to detect overlapping. If overlapping occurs, normal action would be for vehicle that is already inside the roundabout to continue driving at normal speed, and other vehicle to slow down. If there are intelligent agents, roundabout priorities can be rearranged based on type of overlapping, if it can be proven that total waiting times can be reduced. 
The advantage of this approach is that various different combinations can be modeled and examined. In this article, four different cases are identified, for different combinations of cooperative and noncooperative agents in roundabout intersection segment:

- Case 1 is used if there is an IMC and two independent cooperative vehicles. Obviously, here exists largest possibility for improvement, since IMC can provide additional data that vehicles themselves can not, providing even larger speeds above speed limit. In this scenario, if two vehicles have overlapped reservation arrays, IMC can provide speed-up signal for one vehicle, and slowdown signal for another.

- Case 2 is applied if there is an IMC with one cooperative and one non-cooperative vehicle. IMC calculates best scenario based on received reservation matrices and sends an adjustment to cooperative intelligence block. Since it can not affect the noncooperative vehicle, it checks if speedup signal can avoid reservation array overlapping. Otherwise, it sends slowdown signal.

- Case 3 is provided if there are two cooperative vehicles in intersection without IMC. Vehicles need to exchange their reservation matrices and adjust their speed profiles, simultaneously providing collision avoidance for all non-cooperative vehicles that can only be detected with vehicles' sensors.

- Case 4 is used as backup in all other cases (no IMC, none or one cooperative vehicle) and here ordinary roundabout rules apply.

Different cases are depicted in Fig. 7.

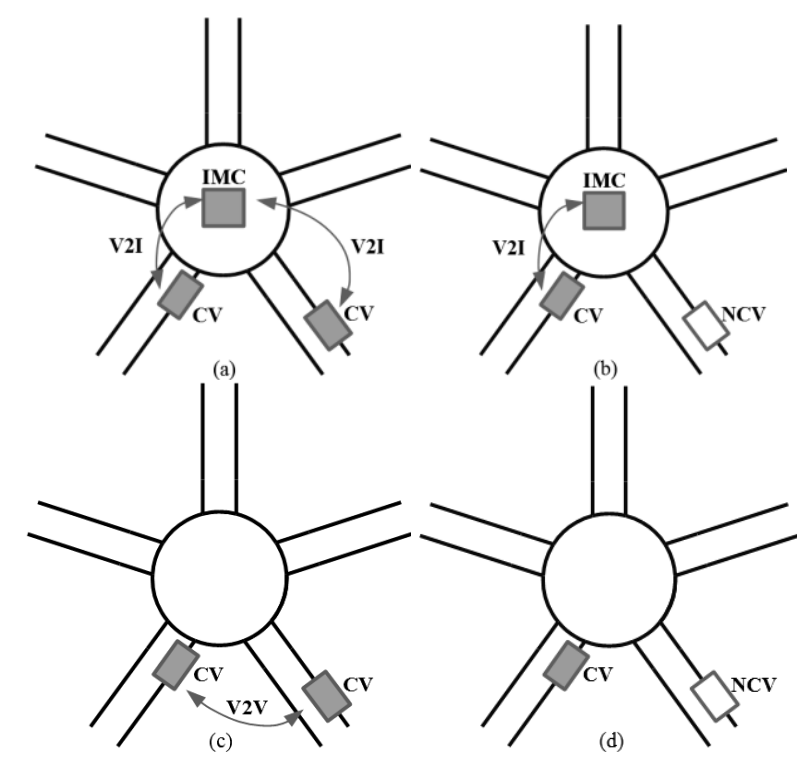

Figure 7. Possible combinations of agents in a roundabout intersection (a) case 1 (b) case 2 (c) case 3 (d) case 4.

Based on these data, intersection manager employs different algorithms in case two vehicles attempt to enter the same roundabout intersection segment at the same time.

If we have both cooperative vehicles and an intersection (Case 1), IMC coordinates with vehicles to make minimal waiting time, as follows:

- Intersection controller compares reservation arrays
- If at current speed limit one vehicle can enter the roundabout before the other vehicle, a zero "speed change" signal is issued to both vehicles

- Controller attempts to correct both vehicles by calculating which vehicle is expected to leave conflicted segment first, so it gets positive speed change signal, while the other gets small negative speed change signal if necessary

- Else larger negative speed change signal is issued to one of the vehicles.

Similar results will be achieved in Case 2, not all options are possible, so slightly worse performance is expected, since one of the vehicles cannot be controlled by IMC (therefore, all scenarios where both vehicle adjust speeds are not possible)

If there are two cooperative vehicles with no IMC (Case 3), two vehicles coordinate to make minimal waiting time, as they calculate which vehicle enters the conflict area first and it has advantage (other vehicle gets negative "speed change" signal.

In Case 4 congestion cannot be improved, since there are not enough intelligent agents to employ coordination. However, fuzzy controller can calculate smooth speed profile based on inter-vehicular distance and relative speed. In this case, normal roundabout traffic rules conditions are used.

\section{Results and discussion}

After implementation of the MATLAB/Simulink model, all four cases were examined in various combinations of entry and exit location, as well as speed limit.

Fig. 8 shows initial reservation arrays for 3 vehicles. As it can be seen, two vehicles have overlapping.

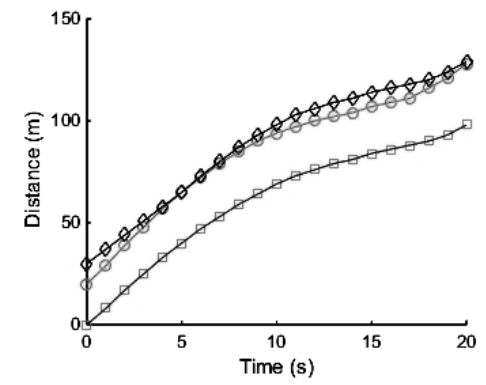

Figure 8. Example of overlapped reservation array.

Fig. 9 shows regular roundabout travel rules - one vehicle slows down while other continues normally. Travel time to $100 \mathrm{~m}$ is $10.45 \mathrm{~s}$ and $14.23 \mathrm{~s}$ for each vehicle, which brings total travel time $24.68 \mathrm{~s}$ for these two vehicles.

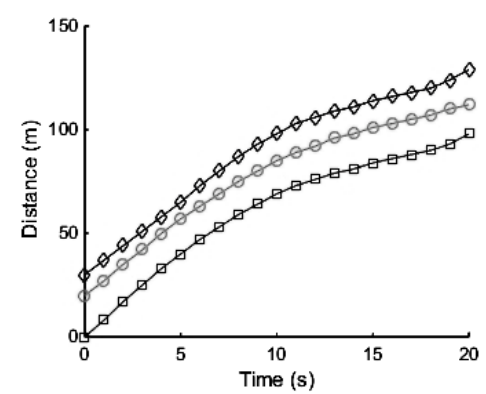

Figure 9. Example of reduced speed for one vehicle (middle line). 
At Fig. 10 we see the best case, where one vehicle speeds up and the other slows down. Travel time to $100 \mathrm{~m}$ is $8.29 \mathrm{~s}$ and $12.37 \mathrm{~s}$ for each vehicle, which brings total travel time $20.66 \mathrm{~s}$, therefore saving more than 4 seconds.

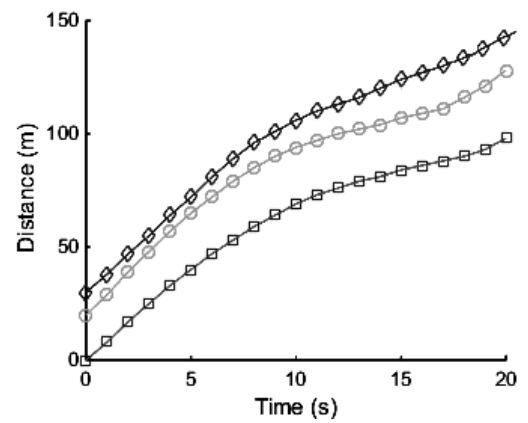

Figure 10. Example of increased speed for one vehicle (top line).

\section{Conclusion and future work}

Simulation has shown that travel time through a roundabout intersection can be reduced. It is shown that system brings improvements in every case - even if there is not intersection controller present.

In future work, we plan to include experiments this case on real-time robotic platform, as well as improving the model (slowing down while entering intersection, more lanes in roundabout, traffic lights, pedestrians, platooning) and improving controlling algorithm. Also, plan is to extend algorithm to coordination with multiple vehicles inside roundabout, so different congestion scenarios can be analyzed.

\section{Acknowledgment}

This paper is supported by the Federal Ministry of Education and Science, Government of Federation of Bosnia and Herzegovina.

\section{References}

1. P. Lipar, J. Kostjansek, "Traffic Calming in Slovenia", 3rd Urban Street Symposium, Seattle, Washington (2007)

2. I. Legac, H. Pilko, N. Subic, "Analysis of traffic safety on roundabout Jadranska Avenue - Avenue Dubrovnik in Zagreb" Conference proceedings Transport, Maritime and Logistics Science, 14th ICTS 2011, Slovenia (2014)

3. J. Ben-Edigbe, A. Abdelgalil, I. Abbaszadehfallah, "Extent of Delay and Level of Service at Signalised Roundabout", International Journal of Engineering and Technology, 2(3), 419 (2012)

4. Y.-J. Gong, J. Zhang, "Real-Time Traffic Signal Control for Modern Roundabouts by Using PSOBased Fuzzy Controller", 2012 IEEE Congress on Evolutionary Computation (2012)

5. T. Tollazzi, M. Rencelj, "Comparative analyse of the two new alternative types of roundabout - turbo and flower roundabout" The Baltic Journal of Road and Bridge Engineering, 9(3), 164 (2014)
6. F. Corriere, M. Guerrieri, D. Ticali, A. Messineo, "Estimation of air pollutant emissions in flower roundabouts and in conventional roundabouts archives of civil engineering", Archives of Civil Engineering, LIX, 2 (2013)

7. S. Jianjun, W. Xu, G. Jizhen, C. Yangzhou, "The analysis of traffic control cyber-physical systems", 13th COTA International Conference of Transportation Professionals (CICTP 2013), (2013)

8. R. Sinha, P.S. Roop, P. Ranjitkar, "Virtual Traffic Lights+: A Robust, Practical, and Functionally Safe Intelligent Transportation System", Transportation Research Record Journal of the Transportation Research Board, (2013)

9. M. Ferreira, P.M. d'Orey, "On the Impact of Virtual Traffic Lights on Carbon Emissions Mitigation", IEEE Transactions on Intelligent Transportation Systems, (2012)

10. H. Ahn, A. Rizzi, A. Colombo, D. Del Vecchio, "Experimental Testing of Semi-autonomous Multivehicle Control for Collision Avoidance at Intersections", IEEE/RSJ International Conference on Intelligent Robots and Systems, (2015)

11. Z. Li, M.V. Chitturi, L. Yu, A.R. Bill, D.A. Noyce, "Sustainability Effects of Next-generation Intersection Control for Autonomous Vehicles", TRANSPORT - Special Issue on Smart and Sustainable Transport, 30(3), 342 (2015)

12. R. Azimi, G. Bhatia, R. Rajkumar, P. Mudalige, "Intersection Management using Vehicular Networks", SAE Technical Paper 2012-01-0292 (2012)

13. Y. Zheng, L. Jin, L. Gao, K. Li, Y. Wang, F. Wang, "Development of a Distributed Cooperative Vehicles Control Algorithm Based on V2V Communication", Procedia Engineering 137, 649 (2016)

14. V. Milanés, J. Pérez, E. Onieva, C. González, "Controller for Urban Intersections Based on Wireless Communications and Fuzzy Logic", IEEE Transactions on Intelligent Transportation Systems, 11(1) (2010)

15. W. Wu, J. Zhang, A. Luo, J. Cao, "Distributed Mutual Exclusion Algorithms for Intersection Traffic Control", IEEE Transactions on Parallel and Distributed Systems, 26(1), 65 (2015)

16. H. Ahn, D. Del Vecchio, "Semi-autonomous Intersection Collision Avoidance through Job-shop Scheduling", arXiv:1510.07026, (2015)

17. H. Ahn, A. Rizzi, A. Colombo, D. Del Vecchio "Experimental Testing of Semi-autonomous Multivehicle Control for Collision Avoidance at Intersections", IEEE/RSJ International Conference on Intelligent Robots and Systems (IROS) (2015)

18. E. Onieva, V. Milanés, J. Villagrá, J. Pérez, J. Godoy, "Genetic optimization of a vehicle fuzzy decision system for intersections", Expert Systems with Applications 39, 13148 (2012)

19. E. Onieva, U. Hernandez-Jayo, E. Osaba, A. Perallos, X. Zhang, "A Multi-Objective Evolutionary Algorithm for the Tuning of Fuzzy Rule Bases for Uncoordinated Intersections in Autonomous Driving", Information Sciences (2015) 
20. M. N. Mladenovic, M. Abbas, "Priority-based Intersection Control Framework for Self-Driving Vehicles: Agent-based Model Development and Evaluation", 2014 International Conference on Connected Vehicles and Expo (ICCVE), 377 (2014)

21. S. H. HosseinNia, B.M. Vinagre, V. Milanés, C. González, "Controller for Urban Intersections Based on Hybrid Automaton", Intelligent Transportation Systems (ITSC), 2010 13th International IEEE Conference, 261 (2010)
22. L.C. Bento, R. Parafita, S. Santos, U. Nunes, "Intelligent Traffic Management at Intersections: Legacy Mode for Vehicles not Equipped with V2V and V2I Communications", IEEE Conference on Intelligent Transport Systems, ITSC2013 (2013)

23. L. C. Bento, R.Parafita, U. Nunes "Intelligent traffic management at intersections supported by $\mathrm{V} 2 \mathrm{~V}$ and V2I communications", IEEE Conference on Intelligent Transportation Systems (2012) 